\title{
Molecular Characterization of Carbapenem-Resistant Acinetobacter baumannii in the Intensive Care Unit of Uruguay's University Hospital Identifies the First $r m t C$ Gene in the Species
}

\author{
Inés Bado, ${ }^{1, *}$ Romina Papa-Ezdra,, Jose F. Delgado-Blas, ${ }^{1}$ Micaela Gaudio,,2 Claudia Gutiérrez, \\ Nicolás F. Cordeiro,' Virginia García-Fulgueiras,' Lucía Araújo Pirez,' Verónica Seija, \\ Julio C. Medina, Gloria Rieppi, Bruno Gonzalez-Zorn,2 and Rafael Vignoli, ${ }^{1, *}$
}

Carbapenem-resistant Acinetobacter baumannii (CRAB) infections are an increasing concern in intensive care units (ICUs) worldwide. The combination of carbapenemases and 16S rRNA-methyltransferases (16S-RMTases) further reduces the therapeutic options. OXA-carbapenemase/A. baumannii clone tandems in Latin America have already been described; however, no information exists in this region regarding the occurrence of 16S-RMTases in this microorganism. In addition, the epidemiology of A. baumannii in ICUs and its associated resistance profiles are poorly understood. Our objectives were as follows: to study the clonal relationship and antibiotic resistance profiles of clinical and digestive colonizing A. baumannii isolates in an ICU, to characterize the circulating carbapenemases, and to detect 16S-RMTases. Patients admitted between August 2010 and July 2011 with a clinically predicted hospital stay $>48 \mathrm{hr}$ were included. Pharyngeal and rectal swabs were obtained during the first fortnight after hospitalization. Resistance profiles were determined with MicroScan ${ }^{\circledR}$ and VITEK2 system. Carbapenemases and 16S-RMTases were identified by PCR and sequencing, and clonality was assessed by pulsed-field gel electrophoresis and multilocus sequence typing. Sixty-nine patients were studied and 63 were diagnosed with bacterial infections. Among these, 29 were CRAB isolates; 49 A. baumannii were isolated as digestive colonizers. These 78 isolates were clustered in 7 pulsetypes, mostly belonging to ST79. The only carbapenemase genes detected were $b l a_{\text {OXA-51 }}(n=78), b_{\text {OXA-23 }}(n=62)$, and bla $a_{\text {OXA-58 }}(n=3)$. Interestingly, two clinical isolates harbored the $r m t C$ 16S-RMTase gene. To the best of our knowledge, this is the first description of the presence of $r m t C$ in A. baumannii.

Keywords: Acinetobacter baumannii, carbapenem resistance, bla $a_{\mathrm{OXA}}, \mathrm{rmtC}$

Introduction

C ARBAPENEM-RESISTANT ACINETOBACTER baumannii (CRAB) infections in critically ill patients are an increasing source of concern in intensive care units (ICUs) worldwide. In 2013, the Centers for Disease Control and Prevention (CDC) categorized multidrug-resistant A. baumannii as a serious threat, responsible in the United States only, for nearly 7,000 infections and 500 deaths per year. ${ }^{1}$ In addition, in 2017 the World Health Organization (WHO) submitted a list of priority bacteria that require urgent de- velopment of new antimicrobials; topping that list was CRAB. ${ }^{2}$ In this regard, aminoglycosides are considered a high-priority group in the critically important antimicrobials list issued by $\mathrm{WHO}^{3}$; due to their synergistic effect, they are often used in combination with $\beta$-lactams to treat MDR gram-negative bacterial infections in healthcare settings, thus constituting one of the last therapeutic options.

Concerning 16S rRNA methyltransferases (16S-RMTases), only a few have been detected in A. baumannii (ArmA, RmtA-RmtH, and NpmA). ArmA is highly prevalent among CRAB strains worldwide, and is mostly associated with the

\footnotetext{
${ }^{1}$ Departamento de Bacteriología y Virología, Facultad de Medicina, Instituto de Higiene, Universidad de la República, Montevideo, Uruguay. ${ }^{2}$ Departamento de Salud Animal y VISAVET, Universidad Complutense de Madrid, Madrid, Spain.

${ }^{3}$ Departamento de Laboratorio Clínico, Área Microbiología, Facultad de Medicina, Hospital de Clínicas, Universidad de la República, Montevideo, Uruguay.

${ }^{4}$ Cátedra de Enfermedades Infecciosas, Facultad de Medicina, Instituto de Higiene, Universidad de la República, Montevideo, Uruguay.

${ }^{5}$ Facultad de Medicina, Unidad de Cuidados Intensivos del Hospital de Clínicas, Universidad de la República, Montevideo, Uruguay.

*These authors share senior authorship for this work.

(C) Inés Bado et al., 2018; Published by Mary Ann Liebert, Inc. This Open Access article is distributed under the terms of the Creative Commons Attribution Noncommercial License (http://creativecommons.org/licenses/by-nc/4.0/) which permits any noncommercial use, distribution, and reproduction in any medium, provided the original author(s) and the source are cited.
} 
carbapenemase gene $b l a_{\text {OXA-23 }}{ }^{4}$ Conversely, RmtB has also been detected in A. baumannii, but only sporadically. ${ }^{5}$ On the other hand, due to the intrinsic broad antibiotic resistance of A. baumannii, carbapenems often constitute the first line of treatment for infections caused by this microorganism. ${ }^{6,7}$ Therefore, 16S-RMTases, which confer resistance to all clinically relevant aminoglycosides, together with carbapenemases, constitute a grave concern for public health worldwide. ${ }^{8}$

Although carbapenemases belonging to each Ambler class have been described in A. baumannii, the most frequently detected are those corresponding to class $\mathrm{D} \beta$-lactamases, such as OXA-23 like, OXA-40 like, OXA-58 like, and OXA143 like. ${ }^{9,10}$ OXA carbapenemases tend to show low levels of identity between different groups; carbapenem resistance conferred by such enzymes is more related to their expression levels than to their hydrolytic profiles, or enzyme-related kinetic properties. ${ }^{11}$ Accordingly, the insertion of different mobile genetic elements, such as ISAbal or ISAba2, upstream of $b l a_{\mathrm{OXA}}$ genes provides strong promoters that enhance the expression levels of the latter. ${ }^{12,13}$

Traditionally, sequence types belonging to clonal complexes $\mathrm{CC} 1, \mathrm{CC} 2$, and $\mathrm{CC} 3$ have been acknowledged as the main lineages harboring oxa carbapenemases, and have been implicated in nosocomial outbreaks in Europe, Asia, and North America. ${ }^{14}$ Nevertheless, the most frequently detected OXA carbapenemase/ST clone tandem in Latin America is OXA-23 in A. baumannii ST79, ST15, or ST25 (none of which belong to any of the aforementioned clonal complexes). ${ }^{15-17}$

We have previously reported that our ICU ventilatorassociated pneumonia (VAP) caused by A. baumannii was preceded by digestive and/or respiratory colonization (DRC) events, and that some of the detected clones were endemic to the ICU. ${ }^{18}$

The aims of this work were to study the clonal relationship of clinical and DRC A. baumannii isolates in the ICU, to analyze the resistance profiles, and to determine the circulating carbapenemases and 16S-RMTases, as well as the clones harboring such enzymes.

\section{Materials and Methods}

\section{Definitions}

Patients were considered community derived if admission to the ICU occurred within $24 \mathrm{hr}$ of being hospitalized, whereas patients were regarded as hospital derived whenever admission to the ICU occurred $>24 \mathrm{hr}$ after being hospitalized.

DRC was considered positive if cultures of pharyngeal or rectal swabs yielded A. baumannii colonies.

Colonization upon admission or ICU-acquired infections were defined according to Medina-Presentado et al. ${ }^{18}$ Briefly, colonization upon admission was considered if $A$. baumannii was isolated from admission samples (pharyngeal and/or rectal); conversely, colonization events were considered ICU acquired if admission samples yielded negative results, but A. baumannii was later recovered from subsequent samples.

\section{Sample processing}

We studied clinical and digestive colonizing A. baumannii isolates obtained from adult ICU inpatients in Uruguay's University Hospital (Hospital de Clínicas Dr. Manuel Quintela). All patients admitted between August 2010 and July 2011 with a clinically predicted hospital stay $>48 \mathrm{hr}$ were included in a successive manner. Clinical samples were submitted to the Clinical Laboratory Department of Hospital de Clínicas and processed routinely (see below). On the other hand, surveillance samples (i.e., DRC samples) were submitted to the Bacteriology and Virology Department for further processing. To avoid redundant patient data, only strains featuring different antibiotic resistance profiles per patient were included in this study.

Bacterial identification and antibiotic susceptibility testing of microorganisms obtained from clinical samples were performed with the VITEK2 system (BioMérieux, Marcy-l'Étoile, France); the microdilution method was performed with the MicroScan ${ }^{\circledR}$ system (Beckman Coulter, Pasadena) following the manufacturer's instructions. We tested susceptibility to the following antibiotics: gentamicin $(\mathrm{N})$, amikacin $(\mathrm{A})$, tobramycin $(\mathrm{T})$, ciprofloxacin $(\mathrm{P})$, ceftazidime (Z), cefepime (F), imipenem (I), meropenem (M), trimethoprim-sulfamethoxazole (X), and colistin (C). In addition, the occurrence of class $\mathrm{A}$ and $\mathrm{B}$ carbapenemases was sought phenotypically by double-disc synergy tests. ${ }^{19}$

Clinical isolates showing resistance to both gentamicin and amikacin were screened for high-level aminoglycosideresistance, in accordance to Hidalgo et al., by their ability to grow on brain heart infusion agar containing both $200 \mathrm{mg} / \mathrm{L}$ amikacin and $200 \mathrm{mg} / \mathrm{L}$ gentamicin. ${ }^{20}$

Susceptibility results were interpreted according to the European Committee on Antimicrobial Susceptibility Testing (EUCAST; www.eucast.org). All nonduplicate clinical isolates for each patient were analyzed.

DRC samples were streaked on MacConkey agar plates (Oxoid Ltd., Basingstoke, UK) supplemented with $1 \mathrm{mg} / \mathrm{L}$ cefotaxime (Libra, Montevideo, Uruguay) and $0.125 \mathrm{mg} / \mathrm{L}$ ciprofloxacin (ION, Montevideo, Uruguay). Up to five putative Acinetobacter spp. colonies per plate were identified by matrixassisted laser desorption ionization-time of flight (MALDI-TOF) mass spectrometry (Bruker, MA). Antibiotic susceptibility testing of DRC isolates was performed with the MicroScan system.

Genes coding for Class A, B, and D carbapenemases were identified by PCR (Table 1). ${ }^{21-23}$ Clinical isolates showing high resistance levels to aminoglycosides were screened by PCR for 16S-RMTase genes (armA, rmtA-rmt H, and $n p m A$ ) (Table 1). ${ }^{24-27}$

Clonality was assessed by pulsed-field gel electrophoresis (PFGE) following digestion with restriction enzyme ApaI (Thermo Scientific, Waltham, MA). Band patterns were analyzed with BioNumerics v.6.6 software (Applied Maths, Sint-Martens-Latem, Belgium) with $2 \%$ tolerance and $0 \%$ optimization. Strains were grouped in clusters on the basis of an $80 \%$ cutoff in accordance with Rafei et al. ${ }^{28}$

Multilocus sequence typing (MLST) was performed on at least one clinical isolate per PFGE profile, following the Pasteur scheme guidelines stated by Diancourt et al. ${ }^{29}$

Numerical variables were analyzed using Fisher's exact test and expressed with their standard deviation; $p$-values $<0.05$ were considered statistically significant. Relative risks (RR) and 95\% confidence intervals (95\% CIs) were calculated using standard methods. Statistics analyses were performed with the SPSS 17.0 software (SPSS, Inc, Chicago, IL).

\section{Results}

Sixty-nine patients (40 men and 29 women) were admitted to the ICU between August 1, 2010, and August 31, 
Table 1. Primers Used to Detect Resistance Genes

\begin{tabular}{|c|c|c|c|c|}
\hline Primer & Sequence $\left(5^{\prime}-3^{\prime}\right)$ & Product size (bp) & $m T$ & Reference \\
\hline$b l a_{I M P}$ & $\begin{array}{l}\text { 5'-AGTCAGGTTTGGCAGATCCGT-3' } \\
\text { 5'-GGTTTAACAAAACAACCACC-3' }^{\prime}\end{array}$ & 684 & 52 & 18 \\
\hline$b l a_{S P M}$ & $\begin{array}{l}5^{\prime} \text {-ATGAACTCACCTAAATCGAGAGCC-3' } \\
\text { 5'-AAACAGCAGTTTCTTCTTGGCC-3' }^{\prime}\end{array}$ & 633 & 60 & 18 \\
\hline bla $_{S I M}$ & $\begin{array}{l}5^{\prime} \text {-TATTCGGCACTTTAAATACCGCG-3' } \\
\text { 5'-GCCACAGTGAAATCGGAGACG-3' }^{\prime}\end{array}$ & 635 & 62 & 18 \\
\hline$b l a_{G I M}$ & $\begin{array}{l}\text { 5'-CTTGTAGCGTTGCCAGCTTT-3' } \\
\text { 5'-TTAATCAGCCGACGCTTCAG-3' }\end{array}$ & 722 & 56 & 18 \\
\hline$b l a_{V I M}$ & $\begin{array}{l}\text { 5'-TAGGAATTCACCATGTTCAAACTTTTGAGTAAGT-3' } \\
\text { 5'-ATAAAGCTTAGCTACTCAACGACTGAGCGA-3' }\end{array}$ & 800 & 55 & 18 \\
\hline$b l a_{N D M}$ & $\begin{array}{l}\text { 5'-GGTTTGGCGATCTGGTTTTC-3' } \\
\text { 5'-CGGAATGGCTCATCACGATC-3' }\end{array}$ & 621 & 56 & 18 \\
\hline$b l a_{K P C-2}$ & $\begin{array}{l}\text { 5'-AACAAGGAATATCGTTGATG-3' } \\
\text { 5'-AGATGATTTTCAGAGCCTTA-3' }\end{array}$ & 915 & 50 & 18 \\
\hline bla $_{\text {OXA-48 }}$ & $\begin{array}{l}\text { 5'-TTGGTGGCATCGATTATCGG-3' } \\
\text { 5'-GAGCACTTCTTTTGTGATGGC-3' }\end{array}$ & 743 & 50 & 18 \\
\hline bla ${ }_{\text {OXA-51 like }}$ & $\begin{array}{l}\text { 5'-TAATGCTTTGATCGGCCTTG-3' } \\
\text { 5'-TGGATTGCACTTCATCTTGG-3' }\end{array}$ & 353 & 52 & 17 \\
\hline bla ${ }_{O X A-23}$ like & $\begin{array}{l}\text { 5'-GATCGGATTGGAGAACCAGA-3' } \\
\text { 5'-ATTTCTGACCGCATTTCCAT-3' }\end{array}$ & 501 & 52 & 17 \\
\hline bla $_{\text {OXA-24 like }}$ & $\begin{array}{l}\text { 5'-GGTTAGTTGGCCCCCTTAAA-3' } \\
\text { 5'-AGTTGAGCGAAAAGGGGATT-3' }\end{array}$ & 246 & 52 & 17 \\
\hline bla $_{\text {OXA-58 like }}$ & $\begin{array}{l}\text { 5'-AAGTATTGGGGCTTGTGCTG-3' } \\
5^{\prime} \text {-CCCCTCTGCGCTCTACATAC-3' }\end{array}$ & 599 & 52 & 17 \\
\hline bla $_{\text {OXA-143 like }}$ & 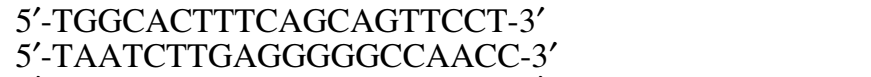 & 149 & 52 & 19 \\
\hline $\operatorname{armA}$ & $\begin{array}{l}\text { 5'-ATTCTGCCTATCCTAATTGG-3' } \\
\text { 5'-ACCTATACTTTATCGTCGTC-3' }\end{array}$ & 315 & 55 & 20 \\
\hline$r m t A$ & $\begin{array}{l}\text { 5'-CTAGCGTCCATCCTTTCCTC-3' } \\
\text { 5'-TTGCTTCCATGCCCTTGCC-3' }\end{array}$ & 635 & 55 & 20 \\
\hline$r m t B$ & $\begin{array}{l}\text { 5'-GCTTTCTGCGGGCGATGTAA-3' } \\
\text { 5'-ATGCAATGCCGCGCTCGTAT-3' }^{\prime}\end{array}$ & 173 & 55 & 20 \\
\hline $\mathrm{rmtC}$ & $\begin{array}{l}5^{\prime} \text {-CGAAGAAGTAACAGCCAAA-3' } \\
5^{\prime} \text {-ATCCCAACATCTCTCCCACT-3' }\end{array}$ & 711 & 55 & 20 \\
\hline$r m t D$ & $\begin{array}{l}\text { 5'-CGGCACGCGATTGGGAAGC-3' } \\
\text { 5'-CGGAAACGATGCGACGAT-3' }^{\prime}\end{array}$ & 401 & 55 & 20 \\
\hline$r m t E$ & 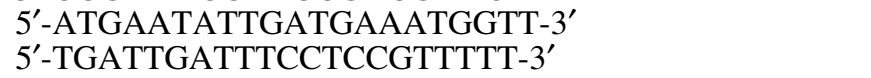 & 818 & 55 & 21 \\
\hline$r m t F$ & $\begin{array}{l}\text { 5'-ACGCATCTGCACCAGATCACC-3' } \\
\text { 5'-GGGCAGGAGCTTCATCAGAA-3' }^{\prime}\end{array}$ & 414 & 61 & This work \\
\hline$r m t G$ & $\begin{array}{l}\text { 5'-AAATACCGCGATGTGTGTCC-3' } \\
5^{\prime} \text {-ACACGGCATCTGTTTCTTCC-3' }\end{array}$ & 251 & 55 & 22 \\
\hline $\mathrm{rmtH}$ & $\begin{array}{l}\text { 5'-AGGTGGAAAAGCAGGCAAG-3' } \\
\text { 5'-CTCAAACCAGGTGGCGTAGT-3' }\end{array}$ & 490 & 55 & This work \\
\hline пртA & $\begin{array}{l}\text { 5'-CTCAAAGGAACAAAGACGG-3' } \\
\text { 5'-GAAACATGGCCAGAAACTC-3' }\end{array}$ & 641 & 58 & 23 \\
\hline
\end{tabular}

$\mathrm{mT}$, melting temperature.

2011. The mean age ( \pm standard deviation) was $52.7 \pm 18.8$ years. The median APACHE II score upon admission was $23.1 \pm 6$, the length of stay in the ICU was $14.7 \pm 12.1$ days, and crude mortality was $33.3 \%$.

Diagnoses upon admission to the ICU were as follows: VAP and respiratory sepsis $(20.3 \%)$, sepsis responding to other causes $(24.6 \%)$, severe trauma or central nervous system (CNS) trauma (24.6\%), CNS infections and acute bacterial meningitis $(8.7 \%)$, stroke $(8.7 \%)$, and other causes (13\%) (Table 2).

Sixty-five ICU-acquired infections were diagnosed: $35.4 \%$ corresponded to VAP, $21.5 \%$ to bacteremia, $16.9 \%$ to purulent tracheobronchitis, $6.2 \%$ to neurosurgical infections,
$4.6 \%$ to urinary tract infections, $4.6 \%$ to catheter-related infections, and $10.1 \%$ to other causes (Table 2).

Eighty-two bacterial agents were recovered from the aforementioned infections; $74.4 \%$ were gram-negative rods and the remaining $25.6 \%$ corresponded to gram-positive bacteria. Among the former, the most frequently detected were as follows: A. baumannii 35.4\%, Pseudomonas aeruginosa $12.2 \%$, and Klebsiella pneumoniae 10.6\%; among gram-positive bacteria, $42.8 \%$ were methicillin-susceptible Staphylococcus aureus (MSSA), $28.6 \%$ were Staphylococcus pneumoniae, $14.3 \%$ were methicillin-resistant $S$. aureus (MRSA), and $14.3 \%$ corresponded to other species (Table 2). 


\section{A. baumannii colonization and infection}

DRC by A. baumannii was detected in 49 patients (71\%), 13 of which $(26.5 \%)$ were already colonized upon admission to the ICU. Regarding the latter, only 1 case of DRC was community acquired, whereas the remaining 12 cases corresponded to acquisitions in different hospital wards.

A. baumannii was recovered from clinical samples in 29 patients, 27 of which were also colonized $(p<0.001, \mathrm{RR}$ : $11.04,95 \%$ CI: $2.31-52.9) ; 25$ of the former corresponded to respiratory samples (14 respiratory secretions, 10 tracheal aspirates, 1 and bronchoalveolar lavage), 2 to central venous catheter samples, 1 to a facial sinus drainage, and 1 to cerebrospinal fluid (Fig. 1). All 29 clinical isolates showed resistance to meropenem (28 of these were also imipenem

Table 2. Demographic, Clinical, and Microbiological Data of the Studied Patients
Gender (male)

Age (SD)

ICU length of stay (SD)

APACHE II score (SD)

Mortality

VAP and respiratory sepsis

Severe trauma

CNS infections and acute

bacterial meningitis

Peritoneal sepsis

Stroke

Soft tissue-related sepsis

CNS trauma

Other causes for sepsis

Cardiac/pulmonary insufficiency

Reanimation cardiorespiratory arrest

Other

ICU-acquired infections (\%)

VAP

Purulent tracheobronchitis

Bacteremia

Urinary tract infections

Catheter-related infections

Neurosurgical infections

Tertiary peritonitis

Skin and soft tissues infections

Other foci

Microorganisms detected (\%)

Gram-negative rods

Acinetobacter baumannii

Pseudomonas aeruginosa

Klebsiella pneumoniae

Other enterobacteria

$H$. influenzae

Gram-positive cocci

Methicillin-susceptible

Staphylococcus aureus

Staphylococcus pneumoniae

Methicillin-resistant $S$. aureus

Enterococcus spp.

Coagulase-negative Staphylococcus
Diagnosis upon admission in the ICU (\%)

Escherichia coli

resistant), cefepime, and ciprofloxacin, whereas 6 isolates displayed resistance to amikacin, gentamicin, and tobramycin, and 3 isolates showed resistance to all the studied antibiotics (except colistin) (Fig. 2). Conversely, all 29 isolates were susceptible to colistin, $69 \%(n=20)$ were susceptible to tobramycin, and $58.6 \%(n=17)$ to gentamicin.

The 78 A. baumanii isolates (29 clinical, 49 colonizing) yielded 20 different resistance profiles, 12 of which included the 29 clinical isolates. Figure 2 depicts the different antibiotypes (ATBtype) detected. The largest profile, ATBtype 1 , featured resistance to amikacin, ciprofloxacin, ceftazidime, cefepime, imipenem, meropenem, and trimethoprimsulfamethoxazole; this profile included $37.9 \%$ and $34.7 \%$ of the clinical and colonizing isolates, respectively. Although ATBtype 1 was found in 6/7 pulsetypes, PTA- V and VI comprised $71.4 \%$ of isolates displaying ATBtype 1 .

Colistin was the only antibiotic active against the 78 studied isolates, constituting the only therapeutic option available, among the tested antibiotics, for 5 isolates (the 3 clinical isolates already mentioned and 2 colonizing isolates). These isolates belonged to ATBtype 5 (Fig. 2).

Interestingly, matching resistance profiles between colonizing and clinical isolates were detected in only five patients, four belonged to ATBtype 1 and the remaining to ATBtype 8 (Fig. 2).

$14(20.3)$

$12(17.4)$

$6(8.7)$

$6(8.7)$

$6(8.7)$

$5(7.2)$

$5(7.2)$

6 (8.7)

2 (2.9)

2 (2.9)

5 (7.2)

23 (35.4)

11 (16.9)

14 (21.5)

3 (4.6)

3 (4.6)

$4(6.2)$

$2(3.1)$

2 (3.1)

3 (4.6)

82

$61(74.4)$

29 (35.4)

10 (12.2)

9 (11.0)

4 (4.9)

7 (8.5)

2 (2.4)

21 (25.6)

9 (11.0)

$6(7.3)$

$3(3.7)$

1 (1.2)

$2(2.4)$

APACHE II, Acute Physiology and Chronic Health Evaluation II; CNS, central nervous system; ICU, intensive care unit; SD, standard deviation; VAP, ventilator-associated pneumonia.

\section{Clonal relationship between colonizing/infecting isolates}

We compared by PFGE 78 A. baumannii isolates, 49 from DRC and 29 from clinical samples. The 78 isolates were clustered in 7 pulsetypes designated PTA-I to PTA-VII. PTA-II, III, V, and VII comprised $91.8 \%$ of the colonizing isolates and $82.8 \%$ of the clinical isolates (Fig. 2). PTA-V was the largest, comprising $47.4 \%$ of isolates recovered from 27 patients $(41.4 \%$ of clinical isolates and $51.0 \%$ of colonizing isolates); PTA-VII was the second largest, including 12 isolates (4 clinical and 8 colonizing) (Fig. 2).

Sixty-three percent of patients $(n=18)$ with A. baumannii clinical isolates were previously colonized by isolates featuring the same pulsetype $(p<0.001, \mathrm{RR}: 4.33,95 \% \mathrm{CI}$ : 2.64-7.12). In 15 of these 18 patients, isolates belonged to PTA-V, II, and VI (Fig. 2).

MLST analysis was performed on 22 clinical isolates belonging to the different pulsetypes. In this sense, 21 corresponded to the ST79, whereas the remaining isolate belonged to a new sequencetype, namely ST958. The latter constitutes a single-locus variant of ST79, since it features allele 6 of gene cpn60 instead of allele 26 .

\section{Detection of resistance genes}

Carbapenemases were sought in all the studied isolates $(n=78)$. All of them harbored the A. baumannii cognate

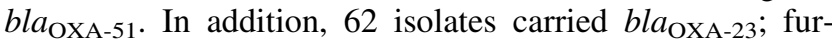
thermore, 3 of such isolates also carried bla $a_{\text {OXA-58. Neither }}$ $b l a_{\mathrm{OXA}-143}$ nor KPC-like or NDM-like class A or B carbapenemases were detected in this study.

Regarding 16S-RMTase genes, rmtC was identified in two of the six clinical isolates displaying high-level aminoglycoside resistance (namely, isolates HCA30 and HCA45). 


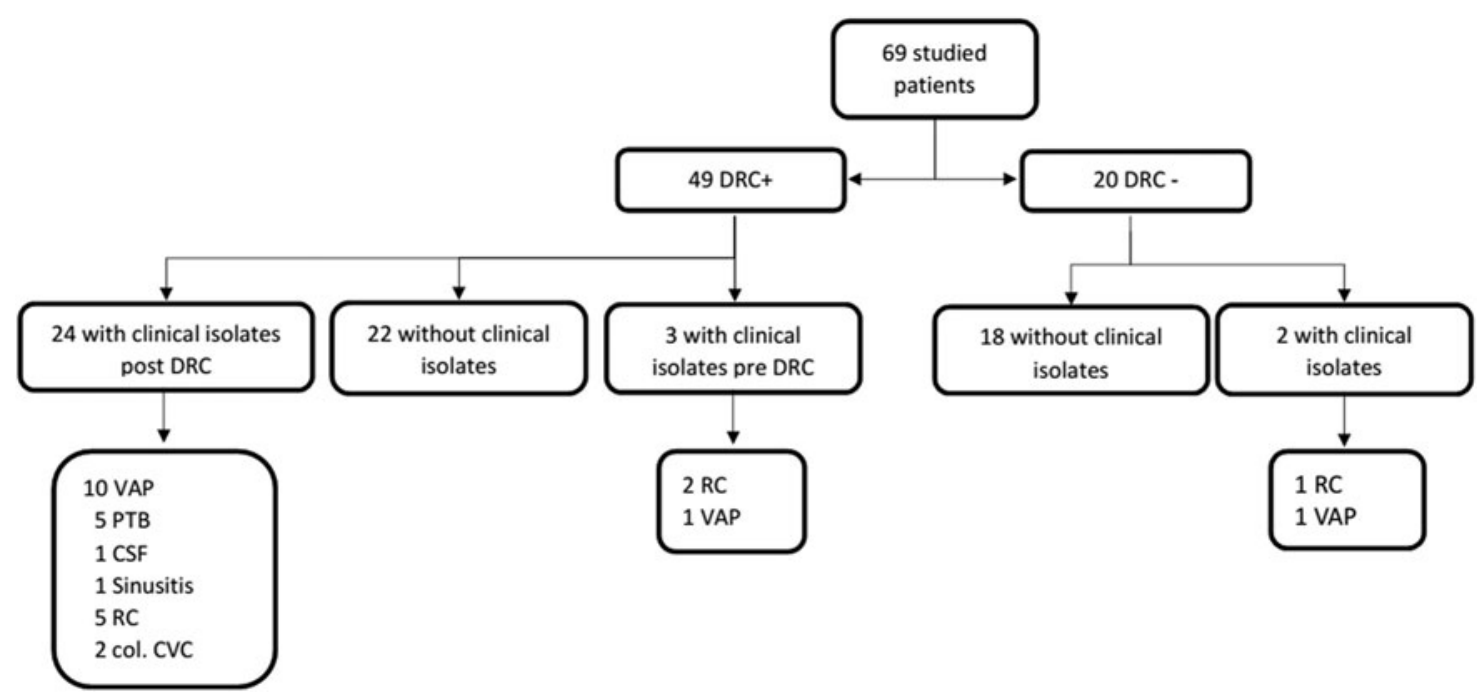

FIG. 1. Distribution of clinical isolates in relationship to the patients' status (i.e., presence or absence of DRC); col. CVC, colonized central venous catheter; CSF, cerebrospinal fluid; DRC, digestive and/or respiratory colonization; PTB, purulent tracheobronchitis; RC, respiratory-colonization ( $\mathrm{RC}$ refers to clinical isolates from respiratory samples [i.e., respiratory secretions or tracheal aspirates], which nevertheless were interpreted as colonization events by the medical staff); VAP, ventilator-associated pneumonia.

\section{Discussion}

CRAB was the main bacterium responsible for infections within the ICU under study, accounting for $35.4 \%$ of the microorganisms identified at the laboratory. Other species included $P$. aeruginosa, K. pneumoniae, and MSSA, constituting $33.2 \%$ of infectious agents. In 2014, Luna et al. reviewed the available information regarding the distribution of gram-negative bacteria circulating in ICUs in South America and the Caribbean, showing a relatively heterogeneous situation between the isolated pathogens. ${ }^{30}$ Most of the reviewed publications indicated that $K$. pneumoniae, $P$. aeruginosa, and $A$. baumannii were among the most frequently detected microorganisms, with A. baumannii fluctuating between $3 \%$ and $50 \%$ of the recovered isolates.

Although the proportion of $A$. baumannii isolates detected in our study is within such range, the impact of carbapenem resistance among such isolates is alarming; in this sense, $100 \%$ isolates were resistant to meropenem, whereas $96.6 \%$ isolates showed resistance to imipenem. Recently, Labarca et $a l$. addressed the issue of carbapenem resistance among clinical isolates of $P$. aeruginosa and A. baumannii in Latin America; their findings showed resistance levels ranging between $7 \%$ and $74 \%$ for $A$. baumannii. ${ }^{31}$

Conversely, the SENTRY program for Latin America (which includes results from Mexico, Brasil, Argentina, and Chile) indicates that among A. baumannii isolates, resistance levels to imipenem and meropenem are $67.8 \%$ and $66.1 \%$, respectively. ${ }^{32}$

The studies mentioned above analyzed isolates obtained from various hospital wards, besides ICUs; taking into consideration only those isolates obtained from patients in the ICU, resistance levels rise to $76.8 \%{ }^{33}$; nevertheless, this resistance level remains below that detected in our center.

MLST analysis of clinical isolates indicated an overwhelming majority of ST79, represented by 21 of the studied isolates. Interestingly, Rodriguez et al. recently reported (in a multicentric study that included isolates from Argentina, Ecuador, Bolivia, Chile, Paraguay, and Uruguay) that the main sequencetype detected in Latin America was ST25, followed distantly by ST79 and ST15. ${ }^{15}$ A. baumannii ST25 strains were detected in 4/6 analyzed countries, including 10 isolates from Uruguay. However, in our study, none of the studied isolates belonged to ST25, thus highlighting the relevance of regional epidemiological differences, even within the same country.

On the other hand, Adams et al. demonstrated recently that the most epidemiologically relevant $A$. baumannii clones, including ST79, harbor on average 33 copies of insertion sequences. ${ }^{34}$ These insertion sequences and transposons confer on A. baumannii a great plasticity, reflected by insertions/deletions as well as changes in the expression of resistance and virulence genes, and metabolic routes. ${ }^{34,35}$ This constant remodeling could account for the great variability in resistance profiles and even in pulsetypes among DRC and clinical isolates. In this regard, in our study, strains displaying PFGE homology levels as low as $62 \%$ were shown to belong to the same sequence type. This genomic plasticity further complicates the analysis of outbreaks caused by such microorganisms.

In our work, $b l a_{\mathrm{OXA}-23}$ was the most frequently detected carbapenemase gene (present in $79.5 \%$ of isolates). Similar data have been reported previously by other authors in the region. ${ }^{15,36}$ On the other hand, the three isolates harboring bla $_{\text {OXA-58 }}$ also carried bla $_{\text {OXA-51 }}$ and bla $_{\text {OXA-23. Finally, in }}$ 16 isolates, the only detected carbapenemase gene was bla $_{\text {OXA-51 }}, 11$ of which were $\mathrm{CRAB}$ and the remaining 5 were susceptible to antibiotics; more studies are required to determine if this gene is under control of a strong promoter, such as the one provided by the insertion of ISAbal. ${ }^{33}$

Rodriguez et al. have recently suggested the occurrence of a progressive substitution of OXA-58 for OXA-23 in A. baumannii isolates. ${ }^{15}$ In this regard, different studies have suggested that the expression of certain $\beta$-lactamases, including 


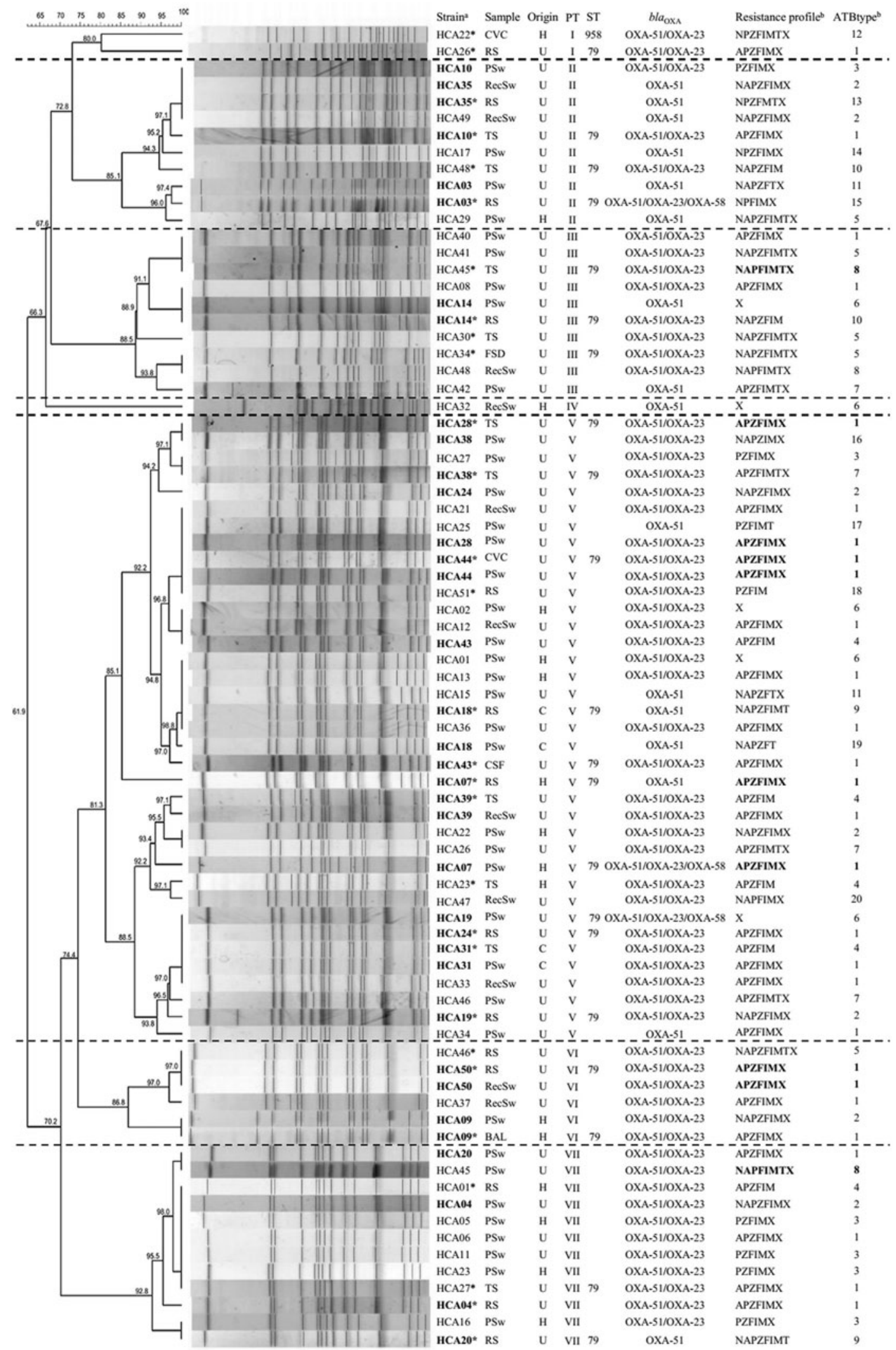

FIG. 2. PFGE profile of the 78 Acinetobacter baumannii isolates obtained from DRC and clinical samples during the study period. Different pulsetypes are separated by horizontal dotted lines. *Clinical sample; ${ }^{\mathrm{a}}$ Isolate numbers in boldface represent those in which both the clinical sample and the DRC sample displayed identical pulsetypes. ${ }^{b}$ Resistance profiles and antibiotpyes in boldface indicate matching resistance profiles between the clinical isolate and the DRC sample. BAL, bronchoalveolar lavage; C, community; CSF, cerebrospinal fluid; CVC, central venous catheter; FSD, facial sinus drainage; H, hospital; PFGE, pulsed-field gel electrophoresis; PSw, pharyngeal swab; PTA, pulse type; RecSw, rectal swab; RS, respiratory secretion; ST, sequence type; TS, tracheal secretion; U, intensive care unit. A, amikacin; F, cefepime; I, imipenem; M, meropenem; N, gentamicin; P, ciprofloxacin; T, tobramycin; X; trimethoprim-sulfamethoxazole; Z, ceftazidime. 
OXA-24, could impose a biological cost on their hosts. ${ }^{37-39}$ Accordingly, the simultaneous expression of several class D carbapenemases could favor the loss, even in presence of carbapenems, of that enzyme which imposes the biggest biological cost on its host.

Further studies are required to determine whether there are fitness variations in those A. baumannii isolates harboring different OXA carbapenemases.

Regarding 16S-RMTases, rmtC was identified in two A. baumannii clinical isolates. To the best of our knowledge, this is the first identification of $r m t C$ associated to this species. These RmtC-producing A. baumannii isolates belonged to PTA-III and sequencetype ST79; both isolates harbored $b l a_{\text {OXA-23 }}$ and bla $a_{\text {OXA-51 }}$ carbapenemase genes. This perilous combination of resistance mechanisms, associated with a successful $A$. baumannii clone, is alarming for the public health. ${ }^{40}$ It would be interesting to determine the potential for mobilization of $r m t C$ to other high-risk clones of $A$. baumannii as well as any fitness cost associated to the expression of $r m t C$; in this regard, these studies could help predict the stability and spreading potential of this gene in this microorganism. ${ }^{41}$

We have already reported the epidemiological aspects of A. baumannii isolates, obtained between 2005 and 2006, from respiratory samples in our ICU. ${ }^{18}$ In this regard, DRC and infections by $A$. baumannii increased from $35.4 \%$ to $71 \%(p<0.001$, odds ratio [OR]: $4.47,95 \%$ CI: $2.44-8.18)$ and $12 \%$ to $24.6 \%$ ( $p=0.016$, OR: $2.53,95 \%$ CI: $1.24-5.20$ ), respectively. We also witnessed a significant increase, although to a lesser degree, in the number of patients already colonized with $A$. baumannii upon admission to the ICU, from $19.4 \%$ to $24.6 \%$ ( $p=0.009$, OR: 3.15 ; CI: $1.36-7.31$ ).

This increase in the incidence of $A$. baumannii along with high levels of resistance to critically important antibiotics calls for continuous clinical and microbiological surveillance, and the implementation of measures aimed at reducing this tendency.

\section{Acknowledgments}

This work was partially supported by grants from Comisión Sectorial de Investigación Científica (CSIC, Montevideo, Uruguay) to R.V. and I.B., as well as grants from Agencia Nacional de Investigación e Innovación (ANII) to I.B.

\section{Disclosure Statement}

No competing financial interests exist.

\section{References}

1. Centers for Disease Control and Prevention. 2013. Antibiotic Resistance Threats in the United States, 2013. CDC Atlanta, Atlanta.

2. World Health Organization. 2017. Global Priority List of Antibiotic-Resistant Bacteria to Guide Reserch, Discovery and Development of New Antibiotics. Available at http://apps .who.int/medicinedocs/documents/s23171en/s23171en.pdf

3. World Health Organization. 2017. Critically Important Antimicrobials for Human Medicine-5th rev. WHO, Geneva. Licence: CC BY-NC-SA 3.0 IGO.

4. Doi, Y., J.-i. Wachino, and Y. Arakawa. 2016. Aminoglycoside resistance: the emergence of acquired $16 \mathrm{~S}$ ribosomal
RNA methyltransferases. Infect. Dis. Clin. North Am. 30: 523-537.

5. Tada, T., T. Miyoshi-Akiyama, Y. Kato, N. Ohmagari, N. Takeshita, N.V. Hung, D.M. Phuong, T.A. Thu, N.G. Binh, N.Q. Anh, T.T.T. Nga, P.H. Truong, P.T. Xuan, L.T.A. Thu, N.T. Son, and T. Kirikae. 2013. Emergence of 16S rRNA methylase-producing Acinetobacter baumannii and Pseudomonas aeruginosa isolates in hospitals in Vietnam. BMC Infect. Dis. 13:251.

6. Montravers, P., A. Harpan, and E. Guivarch. 2016. Current and future considerations for the treatment of hospitalacquired pneumonia. Adv. Ther. 33:151-166.

7. Guan, X., L. He, B. Hu, J. Hu, X. Huang, G. Lai, Y. Li, Y. Liu, Y. Ni, H. Qiu, Z. Shao, Y. Shi, M. Wang, R. Wang, D. Wu, C. Xie, Y. Xu, F. Yang, K. Yu, Y. Yu, J. Zhang, and C. Zhuo. 2016. Laboratory diagnosis, clinical management and infection control of the infections caused by extensively drug-resistant Gram-negative bacilli: a Chinese consensus statement. Clin. Microbiol. Infect. 22(Suppl 1): $\mathrm{S} 15-\mathrm{S} 25$.

8. Hidalgo, L., B. Gutierrez, C.M. Ovejero, L. Carrilero, S. Matrat, C.K.S. A. Saba, Santos-Lopez, D. Thomas-Lopez, A. Hoefer, M. Suarez, G. Santurde, C. Martin-Espada, and B. Gonzalez-Zorn. 2013. Klebsiella pneumoniae sequence type 11 from companion animals bearing ArmAm, DHA-1 $\beta$-lactamase, and QnrB4. Antimicrob. Agents Chemother. 57:4532-4534.

9. Gniadek, T.J., K.C. Carroll, and P.J. Simner. 2016. Carbapenem-resistant non-glucose-fermenting gram-negative bacilli: the missing piece to the puzzle. J. Clin. Microbiol. 54: 1700-1710.

10. Neves, F.C., W.T. Clemente, N. Lincopan, I.D. Paião, P.R. Neves, R.M. Romanelli, S.S. Lima, L.F. Paiva, P.H. Mourão, and V.A. Nobre-Junior. 2016. Clinical and microbiological characteristics of OXA-23- and OXA-143-producing Acinetobacter baumannii in ICU patients at a teaching hospital, Brazil. Braz. J. Infect. Dis. 20:556-563.

11. Poirel, L., T. Naas, and P. Nordmann. 2010. Diversity, epidemiology, and genetics of class D $\beta$-lactamases. Antimicrob. Agents Chemother. 54:24-38.

12. Antunes, N.T., and J.F. Fisher. 2014. Acquired class D beta-lactamases. Antibiotics (Basel) 3:398-434.

13. Pagano, M., A.F. Martins, and A.L. Barth. 2016. Mobile genetic elements related to carbapenem resistance in Acinetobacter baumannii. Braz. J. Microbiol. 47:785-792.

14. Woodford, N., J.F. Turton, and D.M. Livermore. 2011. Multiresistant Gram-negative bacteria: the role of high-risk clones in the dissemination of antibiotic resistance. FEMS Microbiol. Rev. 35:736-755.

15. Rodriguez, C.H., N. Balderrama Yarhui, M. Nastro, T. Nunez Quezada, G. Castro Canarte, R. Magne Ventura, T. Ugarte Cuba, N. Valenzuela, F. Roach, M.I. Mota, N. Burger, G. Velazquez Aguayo, J. Ortellado-Canese, G. Bruni, C. Pandolfo, N. Bastyas, and A. Famiglietti. 2016. Molecular epidemiology of carbapenem-resistant Acinetobacter baumannii in South America. J. Med. Microbiol. 65:1088-1091.

16. Camargo, C.H., M.R. Tiba, M.R. Saes, F.M. Vasconcellos, L.F. Santos, E.C. Romero, and O. Garcia Dde. 2016. Population structure analysis of carbapenem-resistant Acinetobacter baumannii clinical isolates from brazil reveals predominance of clonal complexes 1, 15, and 79. Antimicrob. Agents Chemother. 60:2545-2547.

17. Sennati, S., A.L. Villagran, A. Bartoloni, G.M. Rossolini, and L. Pallecchi. 2016. OXA-23-producing ST25 Acinetobacter 
baumannii: First report in Bolivia. J. Glob. Antimicrob. Resist. 4:70-71.

18. Medina-Presentado, J.C., V. Seija, R. Vignoli, J. Pontet, L. Robino, N.F. Cordeiro, I. Bado, V. Garcia-Fulgueiras, M. Berro, C. Bazet, E. Savio, and G. Rieppi. 2013. Polyclonal endemicity of Acinetobacter baumannii in ventilated patients in an intensive care unit in Uruguay. Int. J. Infect. Dis. 17:e422-e427.

19. The European Committee on Antimicrobial Susceptibility Testing. Guidelines for Detection of Resistance Mechanisms and Specific Resistances of Clinical and/or Epidemiological Importance. Version 2.0, 2017. www.eucast.org

20. Hidalgo, L., K.L. Hopkins, B. Gutierrez, C.M. Ovejero, S. Shukla, S. Douthwaite, K.N. Prasad, N. Woodford, and B. Gonzalez-Zorn. 2013. Association of the novel aminoglycoside resistance determinant RmtF with NDM carbapenemase in Enterobacteriaceae isolated in India and the UK. J. Antimicrob. Chemother. 68:1543-1550.

21. Woodford, N., M.J. Ellington, J.M. Coelho, J.F. Turton, M.E. Ward, S. Brown, S.G. Amyes, and D.M. Livermore. 2006. Multiplex PCR for genes encoding prevalent OXA carbapenemases in Acinetobacter spp. Int. J. Antimicrob. Agents. 27:351-353.

22. Seija, V., J.C. Medina-Presentado, I. Bado, R.P. Ezdra, N. Batista, C. Gutierrez, M. Guirado, M. Vidal, M. Nin, and R. Vignoli. 2015. Sepsis caused by New Delhi metallo- $\beta$ lactamase (blaNDM-1) and qnrD-producing Morganella morganii, treated successfully with fosfomycin and meropenem: case report and literature review. Int. J. Infect. Dis. 30:20-26.

23. Higgins, P.G., M. Lehmann, and H. Seifert. 2010. Inclusion of OXA-143 primers in a multiplex polymerase chain reaction (PCR) for genes encoding prevalent OXA carbapenemases in Acinetobacter spp. Int. J. Antimicrob. Agents. 35:305.

24. Doi, Y., and Y. Arakawa. 2007. 16S ribosomal RNA methylation: emerging resistance mechanism against aminoglycosides. Clin. Infect. Dis. 45:88-94.

25. Davis, M.A., K.N.K. Baker, L.H. Orfe, D.H. Shah, T.E. Besser, and D.R. Call. 2010. Discovery of a gene conferring multiple-aminoglycoside resistance in Escherichia coli. Antimicrob. Agents Chemother. 54:2666-2669.

26. Bueno, M.F.C., G.R. Francisco, J.A. O'Hara, D. de Oliveira Garcia, and Y. Doi. 2012. Coproduction of 16S rRNA Methyltransferase RmtD or RmtG with KPC-2 and CTX-M Group Extended-Spectrum $\beta$-Lactamases in Klebsiella pneumoniae. Antimicrob. Agents Chemother. 57:2397-2400.

27. Fritsche, T.R., M. Castanheira, G.H. Miller, R.N. Jones, and E.S. Armstrong. 2008. Detection of methyltransferases conferring high-level resistance to aminoglycosides in Enterobacteriaceae from Europe, North America, and Latin America. Antimicrob. Agents Chemother. 52:1843-1845.

28. Rafei, R., F. Dabboussi, M. Hamze, M. Eveillard, C. Lemarié, M.-P. Gaultier, H. Mallat, R. Moghnieh, R. Husni-Samaha, M.-L. Joly-Guillou, and M. Kempf. 2014. Molecular analysis of Acinetobacter baumannii strains isolated in Lebanon using four different typing methods. PLoS One 9:e115969.

29. Diancourt, L., V. Passet, A. Nemec, L. Dijkshoorn, and S. Brisse. 2010. The population structure of Acinetobacter baumannii: expanding multiresistant clones from an ancestral susceptible genetic pool. PLoS One 5:e10034.

30. Luna, C.M., E. Rodriguez-Noriega, L. Bavestrello, and M. Guzman-Blanco. 2014. Gram-negative infections in adult intensive care units of latin america and the Caribbean. Crit. Care Res. Pract. 2014:480463.
31. Labarca, J.A., M.J. Salles, C. Seas, and M. GuzmanBlanco. 2014. Carbapenem resistance in Pseudomonas aeruginosa and Acinetobacter baumannii in the nosocomial setting in Latin America. Crit. Rev. Microbiol. 42:276-292.

32. Gales, A.C., M. Castanheira, R.N. Jones, and H.S. Sader. 2012. Antimicrobial resistance among Gram-negative bacilli isolated from Latin America: results from SENTRY Antimicrobial Surveillance Program (Latin America, 2008-2010). Diagn. Microbiol. Infect. Dis. 73:354-360.

33. Castilho, S.R.A., C.S.M. Godoy, A.O. Guilarde, J.L. Cardoso, M.C.P. Andre, A.P. Junqueira-Kipnis, and A. Kipnis. 2017. Acinetobacter baumannii strains isolated from patients in intensive care units in Goiania, Brazil: molecular and drug susceptibility profiles. PLoS One 12:e176790.

34. Adams, M.D., B. Bishop, and M.S. Wright. 2016. Quantitative assessment of insertion sequence impact on bacterial genome architecture. Microb. Genom. 2:e000062.

35. Li, H., F. Liu, Y. Zhang, X. Wang, C. Zhao, H. Chen, F. Zhang, B. Zhu, Y. Hu, and H. Wang. 2015. Evolution of carbapenem-resistant Acinetobacter baumannii revealed through whole-genome sequencing and comparative genomic analysis. Antimicrob. Agents Chemother. 59:1168-1176.

36. Dias, V.C., C.G. Diniz, A.C. Peter, A.N. Bastos, V.Q. Bastos, L.Q. Bastos, and V.L. Da Silva. 2016. Epidemiological characteristics and antimicrobial susceptibility among carbapenem-resistant non-fermenting bacteria in Brazil. J. Infect. Dev. Ctries. 10:544-553.

37. Cordeiro, N.F., J.A. Chabalgoity, L. Yim, and R. Vignoli. 2014. Synthesis of metallo-beta-lactamase VIM-2 is associated with a fitness reduction in Salmonella enterica serovar Typhimurium. Antimicrob. Agents Chemother. 58:6528-6535.

38. Cordeiro, N.F., L. Yim, L. Betancor, D. Cejas, V. GarcíaFulgueiras, M.I. Mota, Varela, G.L. Anzalone, G. Algorta, G. Gutkind, J.A. Ayala, J.A. Chabalgoity, and R. Vignoli. 2013. Identification of the first blaCMY-2 gene in Salmonella enterica serovar Typhimurium isolates obtained from cases of paediatric diarrhoea illness detected in South America. J. Glob. Antimicrob. Resist. 1:143-148.

39. Fernandez, A., A. Perez, J.A. Ayala, S. Mallo, S. RumboFeal, M. Tomas, M. Poza, and G. Bou. 2012. Expression of OXA-type and SFO-1 beta-lactamases induces changes in peptidoglycan composition and affects bacterial fitness. Antimicrob. Agents Chemother. 56:1877-1884.

40. Hopkins, K.L., J.A. Escudero, L. Hidalgo, and B. Gonzalez-Zorn. 2010. 16S rRNA methyltransferase RmtC in Salmonella enterica serovar Virchow. Emerg. Infect. Dis. 16:712-715.

41. Gutierrez, B., J.A. Escudero, A. San Millan, L. Hidalgo, L. Carrilero, C.M. Ovejero, A. Santos-Lopez, D. ThomasLopez, and B. Gonzalez-Zorn. 2012. Fitness cost and interference of Arm/Rmt aminoglycoside resistance with the RsmF housekeeping methyltransferases. Antimicrob. Agents Chemother. 56:2335-2341.

$$
\begin{array}{r}
\text { Address correspondence to: } \\
\text { Rafael Vignoli, MD, PhD } \\
\text { Departamento de Bacteriología y Virología } \\
\text { Facultad de Medicina } \\
\text { Instituto de Higiene } \\
\text { Universidad de la República } \\
\text { Montevideo 11600 } \\
\text { Uruguay }
\end{array}
$$

E-mail: rvignoli@higiene.edu.uy 